\title{
Entrelacs
}

Cinéma et audiovisuel

\section{Du corps à l'espace et de l'espace au corps}

Edwige Armand

\section{OpenEdition}

\section{Journals}

Electronic version

URL: http://journals.openedition.org/entrelacs/5962

DOI: 10.4000/entrelacs.5962

ISSN: 2261-5482

\section{Publisher}

Éditions Téraèdre

\section{Electronic reference}

Edwige Armand, « Du corps à l'espace et de l'espace au corps », Entrelacs [Online], 17 | 2020, Online since 01 July 2020, connection on 03 July 2020. URL : http://journals.openedition.org/entrelacs/5962 ; DOI : https://doi.org/10.4000/entrelacs.5962

This text was automatically generated on 3 July 2020.

Tous droits réservés 


\title{
Du corps à l'espace et de l'espace au corps
}

\author{
Edwige Armand
}

1 La vision, comme tous les sens humains, est un sens actif et cognitif à des niveaux divers. Il y a toujours acte de participation dans la lecture et l'écriture du perceptible.

2 Avant qu'une lecture de signes iconiques, symboliques ou indiciels puisse émerger, le monde physique perçu comme indépendant de soi doit s'instaurer. Cette instauration d'un monde physique est possible par l'empathie où l'enfant développe la capacité de se mettre à la place d'autrui ${ }^{1}$. Le milieu primordial s'inaugure comme indépendant lorsque l'enfant est capable d'imaginer le regard de l'autre. Le détachement d'un monde transcendant est au départ affectif et qui se développer par la reconnaissance des proches nous dit Axel Honneth, dans La réification'. C'est par l'Autre que le monde apparaît comme monde situé à l'extérieur de soi. En même temps que le monde s'externalise, le sujet s'internalise dans une relation de réciprocité par jeu de projection et d'introjection. La reconnaissance d'un soi, d'un monde est intrinsèquement liée à un partage intersubjectif où l'autre nous désigne comme sujet. Avec l'acquisition du langage, l'espace du monde s'inscrira dans une structuration symbolique, articulée par une temporalité linéaire sous-jacente à la pensée liée à l'écriture et dans laquelle se nouera un mode du visible. La pensée articulée à l'écriture selon J. Derrida ${ }^{3}$ est ce qui va créer l'ordonnancement phénoménal dans une logique linéaire et artificiellement cohérente, créant un écart entre l'immédiateté d'un temps qui se fait et se défait simultanément et une couche surajoutée à l'événement. L'événement vécu se réalise en partie par la pensée articulée à la langue. Par la langue nous passons de l'infans inarticulé du cri à une articulation spatio-temporelle arbitraire. La spatialisation du temps par la langue et la sédimentation culturelle des signes permettront de mettre à l'écart le réel tout en construisant un rapport inachevé au présent. La mise en espace du monde par le langage et les signes culturels conjugueront l'inextensivité du temps et des sensations par à une extensivité perceptive; le monde apparaît sous une forme stabilisée dans laquelle le corps peut se situer et se séparer. Cette séparation entre un monde extériorisé et un corps sensible affectif permet d'accéder à la subjectivité tout 
en subsumant l'interpénétrabilité d'un corps et d'un monde. Merleau-Ponty ${ }^{4}$ critiqua cette certitude naïve d'un monde indépendant puisque le corps et le monde sont bâtis autour de la perception qui ne se trouve que dans le corps. Cette vision séparatrice entre un monde et un soi, tant nécessaire pour considérer autrui et se subjectiviser en retour produit l'erreur de percevoir qu'un monde extérieur préexisterait à notre perception. Merleau-Ponty dans Le visible et l'invisible nous rappelle qu'il n'y a pas de monde brut, mais un monde élaboré. Autrement dit, il n'y aurait pas d'intermonde ni de partage sensible et intersubjectif du monde, chacun étant condamné à ne vivre que sa vie et les autres n'étant que des autres soi-même. Cette production du monde sensible et visible par le corps, où le corps et le cerveau ne pourraient bien être qu'image à leur tour pour G. Deleuze ${ }^{6}$, et où l'image n'est pas non plus dans le cerveau, le cerveau étant lui-même image, rend indéniablement active la visibilité de l'espace. L'espace est ce que je projette, mais qui en retour me permet de m'introjecter.

Dans la relativité d'un voir et d'un corps, pourtant un monde partageable s'est modélisé. En effet, l'ensemble de nos techniques de vision (dont le langage fait partie) a permis de construire un accord commun du visible, accentuant l'idée qu'un monde était comme déjà-là et en dehors de nous, développant le leurre d'une vision indépendante du corps. Les sciences et les techniques ont accentué en plus du langage, cette pensée d'un monde perçu comme objectif et externe à soi. La distinction entre sujet et objet opérée par nos techniques de visions et nos techniques cognitives ont accentué une réification du visible que le regard affecté par l'autre avait déjà permis d'amorcer.

4 Se départir dès lors du langage et d'une certitude d'un monde visible, revenir à une proximité absolue permettrait pour Merleau Ponty ${ }^{7}$, de revenir à l'épaisseur de la chair du monde et d'une conscience d'être créateur de monde. Prendre appui sur le corps dans une conscience, une corporéité exacerbée et originale reviendrait à explorer l'être possible. Faire l'expérience de son corps, c'est devenir un corps co-émergeant avec un monde. Notre présence et notre capacité à être là en totalité sont vitales pour passer du devant au dedans, du dedans au-devant, dans une conscience qui en saisit l'impossibilité des termes. Dans la chute du temps ou la revivance dans la durée pure non spatialisée, c'est alors un corps qui peut s'esquisser, rompant l'ordonnancement linéaire qui construit le sens. Cette possibilité de refonte du sens est le terrain de l'artiste qui développe un savoir qui peut mettre au jour l'indifférence des sens et des mondes perçus allant de pair avec la relativité du monde et celle des découpages des corps. Avant d'être un mode de connaissances, la création présuppose l'internalisation d'un savoir, en termes clairs et distincts. L'acte de création serait comme une réaction au savoir, comme une prise de conscience, presque épidermique, en réaction aux acquis symboliques, imaginaires, aux préjugés spatio-temporels ainsi qu'à nos habitudes cognitives et perceptives.

5 Les arts permettent de restituer un sens "sauvage ", dans la précarité du temps où la lecture du monde se défait des signes surnotés constitutifs du visible interprété.

$6 \quad$ Les arts produisent une libération du regard proposant un jeu entre les langages articulés eux-mêmes, entre les langages et les choses, entre les choses et les êtres, entre l'être et lui-même. C'est dans l'écart immiscé entre le monde et un corps que peuvent s'articuler d'autres formes de visibilité et de présence. Parce que l'art ne relève pas nécessairement de la pensée articulée à la langue que l'évènement peut surgir dans ce qu'il a de plus radicalement autre, dans une brutalité inanalysée et non catégorisée 
pour reprendre les termes de Peirce ${ }^{8}$. C'est dans la proximité et la confrontation avec un réel, que la subjectivité de l'artiste peut s'inaugurer, réarticulant des signes et des rythmes sensoriels singuliers non soumis à l'arbitraire des sens. C'est ce fragment de monde possible que l'artiste propose au regard du spectateur. Spectateur invité à se départir d'un sens surimposé par la culture et le regard de l'Autre. C'est une manifestation autre d'un monde adressée en fin de compte à autrui pour qu'elle devienne partageable. Dans le fragment de réel que propose l'art au spectateur, celui-ci redécouvre la puissance d'instauration d'un sens, dans une singularité qu'il peut accomplir. En réarticulant l'espace et le temps du visible, du sonore, le spectateur se révèle dans une corporéité où les rythmes sensibles rejouent de nouvelles présentations affectives d'un monde oublié comme création du corps.

7 L'art doit venir nous déranger dans notre quiétude, dans l'évidence du tout est normal, dans le fait de considérer que tout est réglé. Penser c'est chercher à signifier. Non pas seulement signifier le monde, mais se signifier soi-même dans un choix privilégié investi de significations particulières. Les arts dans leurs ensembles doivent offrir au spectateur une réactualisation active de la chair des concepts et des significations.

Or, aujourd'hui, nous sommes loin d'une telle démarche dans l'industrie audiovisuelle (ou bien encore dans un marché de l'art qui recherche la séduction du public en le flattant dans ce qui est connu et évident), tout comme nous sommes loin de scénarios temporels et spatiaux qui chercheraient la possibilité de se dessaisir du sens et d'une déliaison du temps qui y est intriqué.

9 Peu de dispositifs de vision aujourd'hui (diffusés à grande échelle) proposent des expériences nous permettant de recouvrer une expérience pré-verbale ou a-parlante. Les dispositifs audiovisuels de l'industrie culturelle créent au contraire des narrations et des récits ne faisant que rassurer le spectateur d'un sens d'un monde ordonné et signifié linéairement. Comme l'énonce Marie-José Mondzain', l'intensification de la vitesse des images, le rythme sur-saturé du visuel et du sonore ne permet aucune symbolisation possible et n'assure aucune intégration du sujet dans l'espace audiovisuel. Pourtant, regarder revient à se regarder, à se projeter dans l'espace visuel, regarder permet de s'inscrire dans un monde procurant la sensation d'exister, dans toute l'inquiétude que cela suppose.

10 La saturation sonore et visuelle par les industries de l'image assujettit le regard et l'ouie du spectateur à un trop-plein sensoriel et à la violence d'une hypercertitude du monde. En créant un sensible saturé et un imaginaire mondialement partagé et communiqué, les industries du visible, font taire la parole du sujet non pas pour la mettre sous silence, mais pour l'exclure de toute possibilité de subjectivation que permettrait un tempo de l'écart ou une arythmie entre l'image et le sonore, ou entre le temps et l'espace. Le spectateur n'est pas interpellé comme sujet, tout est mis à disposition de son regard, aucun vide provoquant l'hésitation du sens comme du monde n'est convoqué. Plutôt que de proposer une expérience du visible spatiale et temporelle dans une écriture décollectivisante, les expériences cinématographique (grand public) et télévisuelle ne fait que reproduire un cadre perceptif consensuel et déjà domestiqué. Trop peu de scénarios pensent l'auctorialité et l'autorité du spectateur comme puissance de composition du sens.

11 Pourtant qu'il s'agisse de la perspective, de la photographie, de la vidéographie, de la vidéo $360^{\circ}$, de la VR, du jeu vidéo, dans tous les cas le spectateur peut-être potentiellement acteur, auteur, car l'interprétation du sens et des signes du monde lui 
revient. Il ne peut que l'être si le dispositif lui permet bien d'écrire le sens des signes et produire à partir des symboles, des indices et des icônes d'autres formes de pensées disjointes de la culture et de la langue.

Comme énoncé précédemment la vision est avant tout prise de position et organisation du monde qui la rend virtuellement active par une corporéité. Elle reste passive lorsqu'elle ne se sépare pas des automatismes de pensées et des mécanismes perceptifs, induits notamment par la pensée linéaire articulée à la langue. L'une des vocations de l'art est bien de nous ouvrir à l'inanité du sens du monde afin d'inviter chacun à une pensée créatrice où surgiraient d'autres horizons. Lorsque le sens n'est pas prédéfini ni orienté (vers un sens classique d'écriture spatio-temporel), il incombe alors au spectateur de faire acte de création, d'augmenter les possibilités de sens, en d'autres termes de se subjectiviser. En réinventant des rythmes spatio-temporels et une lecture des signes, d'autres manières de présenter le phénomène peuvent émerger. Le temps linéarisé construit une trame artificielle d'un temps qui ne possède pourtant aucun sens, aucune orientation, aucune direction et qui est dépourvu de toute téléologie. C'est dans cette illusion temporelle que s'écrit un sens surimposé. Délinéariser le temps, déspatialiser le phénomène, le rendre non téléologique, permet le jaillissement infini des possibles où le sens revient à l'autorité du spectateur désirant. L'interactivité réelle, celle qui donne la possibilité au spectateur de se charger de la lecture du sens de l'événement visible ne se réalisera que par l'effacement d'un sens prédonné, où le spectateur acquiert progressivement une écriture singulière des données d'un monde qu'il participe à produire. Si aucune place ne lui est laissée dès lors, le spectateur demeure dans une position de sujétion. À l'origine, l'interactivité suppose un échange, un effet de boucle rétroactive. Cela signifie que le spectateur influe sur le déroulement de l'œuvre et que l'œuvre en retour agit sur le spectateur. De très rares œuvres présentent cette caractéristique. La plus célèbre est celle de M.H Tramus et M.Bret dans Danse avec moi ou la Funambule (2001); il s'agit d'une œuvre de la seconde interactivité où le programme évolue par des réseaux de neurones qui sont dotés d'apprentissage et intègrent les gestes des spectateurs. Dans les œuvres évolutives comme Quorum Sensing (2002) de Chu-Yin Chen, les créatures artificielles évoluent par les mouvements des spectateurs grâce à des algorithmes génétiques. Les jeux vidéo peinent quant à eux à développer des scénarios non prédéterminés, enfermant le spectateur dans des choix limités et reproductibles, sans que celui-ci puisse réellement avoir une incidence sur le déroulement scénaristique pensé par arborescence basé ainsi le modèle de l'arbre où les possibilités ne sont que limitées et pré-tracées. Quelques œuvres utilisent ce que l'on nomme du temps souple, initié notamment par les recherches développées par Thierry Besche au GMEA et dans lesquelles les paramètres des scénarios peuvent être modulés en fonction des mouvements des acteurs. Ici, les scénarios s'adaptent au temps $\mathrm{du}$ corps des spectateurs ou acteurs. Dans la pièce Le courage (2013), de Jean-Léon Pallandre, le fade in fade out de la lumière et du son sont fonction de la vitesse de déplacement de l'acteur. D'autres œuvres encore utilisent des vocabulaires visuels ou sonores qui s'actualisent de manière aléatoire en fonction là aussi de l'activité du spectateur. C'est par exemple l'œuvre de Mathieu Chamagne, Aperture (2014). Une majorité d'œuvres interactives, malgré la volonté d'intégrer le spectateur en le laissant penser qu'il agit sur l'œuvre, restent toutefois dans des scénarios actualisés par le principe de l'aléatoire où une version possible de l'œuvre émerge. Ce genre d'interactivité permet de faire varier la réalisation de l'œuvre et d'avoir une diversité de combinaisons scénaristiques. En revanche, le spectateur n'a finalement que peu de 
choix et de maîtrise sur le devenir de la pièce, et subit le déterminisme-aléatoire sans avoir un impact décisionnaire sur le résultat.

Ainsi, sous couvert de nouveauté incluant le terme d'interactivité, l'auteur propose toujours une intention et le spectateur ensuite dispose du sens, en fonction de la liberté d'interprétation que permet d'ouvrir l'œuvre. Pourtant nos techniques ont toutes un potentiel créateur d'écriture spatio-temporelle, visuelle et sonore originale qui permettrait au spectateur de se subjectiviser en se départissant d'un sens donné d'avance, et lui permettrait d'être auteur de la lecture de l'événement. Dans un scénario audiovisuel classique, le spectateur discrimine les éléments qui font sens dans l'espace visuel, le tempo du temps, suit généralement la succession linéarisée d'images. Les auteurs de la nouvelle vague, ont réussi à inventer d'autres écritures spatiotemporelles et se sont imposés malgré l'hégémonie du visible et du narratif des industries culturelles. Cela est vrai également pour les artistes de l'art vidéo, comme Gary Hill ou bien encore les Vasulka. À chaque invention technique, des possibilités immenses de créations s'ouvrent, mais l'on observe trop souvent qu'aucune expérience sensible et narrative différente n'est finalement proposée. Avec la vidéo $360^{\circ}$ ou la VR, nous sommes face à cette même problématique d'écriture. Alors qu'il est possible aujourd'hui d'imaginer dans une même image une multiplicité d'événements qui coexisteraient et des séries simultanées de réalités qui émergeraient sur un même plan, nous retombons dans la majorité des cas, dans des scénarios linéaires induisant un sens historique (c'est-à-dire revenant encore à une histoire) à l'adresse du spectateur soumis encore, à la narration. Narration soumise à la pensée linéarisée dont la langue est le soubassement. Langue qui supplée l'originalité de l'événement et éloigne la réalisation de sa subjectivité en masquant la faillite de la temporalité et évite de penser l'écart entre le réel et la réalité (que s'exerce à combler le sens coordonné par la langue). Faillite et écart permettant pourtant au sujet de créer un sens singulier dans une projection désirante et désirée dans l'éphémérité d'un monde.

14 L'immédiateté du temps, dans ce qu'elle a de plus brutale, permet de rompre nos habitudes sensori-motrices et perceptives. C'est ce même temps qui est divisé par l'écriture, constituant les instants $t$, les arrêts virtuels dont parlait Bergson ${ }^{10}$ puis Deleuze $^{11}$, qui sont ensuite linéarisés par une succession rendant cohérent un monde qui ne l'est pas. Doit-on alors rassurer encore le spectateur dans une même organisation spatio-temporelle et historique, ou bien doit-on le conduire à l'expérience du sans bord, du sans sens, de la marge du monde que l'art peut en puissance permettre de réaliser?

Certains metteurs en scène aujourd'hui cherchent la réinvention de l'articulation spatio-temporelle en se détachant de la trame narrative. Par les matériaux du plateau, ils cherchent par l'ensemble des signes (plastiques, iconiques, symboliques, indiciels) à rouvrir le sens d'une linéarité qui n'est pas innée, mais héritée d'une longue histoire culturelle et d'une conception euclidienne d'un espace-temps. Avec les metteurs en scène appelés les écrivains de plateau, le mot, la lettre, le texte deviennent périphériques et acquièrent une égalité de valeur avec la lumière, le son, les corps. Ces écrivains réussissent en multipliant non seulement les niveaux de sens (ce qui est inhérent aux œuvres d'art puisque toute œuvre est polysémique) et simultanément à mettre sur un même plan des séries de phénomènes a priori disjoints dans lesquels la cohérence et persistance d'un sens n'est pas donnée. Ils nous plongent dans une indétermination, une indécidabilité visuelle, sonore, textuelle, où il impute au 
spectateur le pouvoir de décider d'un sens ou au contraire de s'accommoder au nonsens, au hors-langue. Dans cette multiplicité simultanée, où la vision de l'espace-temps devient indocile alors le corps peut esquisser d'autres frontières fragiles d'un corps en perpétuelle projection. L'espace du sens ouvert, la projection d'autres limites et frontières corporelles-cognitives deviennent possibles.

Pour conclure Nietzsche ${ }^{12}$ critiquait le fait que la modernité, prétendait de manière mensongère rompre avec un passé qu'elle prolongeait de manière déguisée. Pour lui, la modernité recycle l'ancien en tentant de donner un goût de nouveauté.

Notre persistance dans l'écriture remonterait selon Derrida ${ }^{13}$ à la naissance de l'agriculture avec l'exploitation de l'espace par les sillons. D'autres manières de vivre le monde, de l'affecter et de s'affecter existent et ont existé. C'est le cas de nos ancêtres Homo Sapiens. Il y a 36000 ans l'espace et le temps n'étaient pas articulés sous la domination de la ligne et ne possédaient pas l'individuation que nous concevons. La lecture du monde était faite de fuite, de trace, de surgissement, de souffle, de rythme, de respiration, de stase, de détour, de mouvement, dans une vision polymorphique et polychronique.

18 La pensée subordonnée à l'écriture est bien une forme de dépossession subjective, d'artificialisation d'un sens qu'on nous impose de partager et liée à la mondialisation d'une domination affective et sensitive. Le mot, la lettre condamne l'événement à une répétition, nous écartent de la variabilité d'un temps vivant en y insérant des états stables, et nous privent de l'originalité de l'événement. Peut-être dès lors, faudrait-il créer des scénarios sauvegardant le silence d'un monde et cherchant à recouvrer le rapport mutique de l'événement comme le propose Pascal Quignard, pour qui, tout acte d'écriture ou de parole sépare et exclut.

Loin de rassembler, l'écriture, sous l'apparent partage d'un monde saturé par le lisible et le dicible, nous plie dans l'imposture d'une pensée collective illusoire. C'est alors dans le chaos apparent d'un mode du voir et du dire, que peut se réinventer l'imaginaire et le discours du sujet. Les modes d'écritures du visible, du sensible ne proposant pas d'historicisation, permettraient au sujet de loger des significations singulières élaborées par l'effort de sa pensée créatrice. L'inconfort proposé au regard et à la parole par ce genre d'écriture permet par la présence du mutisme qu'ils provoquent, une amélioration d'un partage du sensible en devenir, où les rapports de parole, d'imaginaire compris comme écart au réel, peuvent se révéler dans leur puissance de réalisation. Écart permettant également de repenser sa subjectivité en réinventant un temps et un espace qui nous soient propres dans une solitude exempte du regard de l'Autre. C'est par une redéfinition de son propre regard, qui dépend d'une articulation spatio-temporelle, que peuvent croître notre singularité et notre subjectivité. Il nous est possible de reconquérir dans des limites repoussées d'autres lectures du monde jamais foulé.

En réinventant des dispositifs d'écriture de l'image et du son, c'est le vacillement du vide qui est offert au spectateur et celui-ci peut restructurer le réel par la médiation de l'imaginaire et du symbolique, jouant ainsi sur la plasticité des réalités et la diversité de pensées. En modifiant son regard, c'est aussi l'Autre désigné par ce nouveau regard qui lui permet de se réinventer en luttant contre sa consommation et sa réification, en lui proposant une errance des regards, dans un rien où l'on peut s'exercer à élancer un je que l'on sait fragile pour assumer un présent et modifier ainsi les horizons des événements. 


\section{NOTES}

1. Axel Honneth, La réification: Petit traité de Théorie critique, Paris, Poche, 2007.

2. Ibid.

3. Jacques Derrida, De la grammatologie, Paris, Les éditions de Minuit, 1967.

4. Maurice Merleau-Ponty, Le visible et l'invisible, Paris, Gallimard, 1964.

5. Ibid, p. 308.

6. Jean-Clet Martin, Arnauld Villani, Problème, Le vocabulaire de Gilles Deleuze, in Robert Sasso, Arnaud,Villani (dir.), Les Cahiers de Noesis, n³, Printemps, 2003, p. 293.

7. Maurice Merleau-Ponty, Le visible et l'invisible, op. cit.

8. Charles Sanders Peirce, Écrit sur le signe, Paris, Seuil, 1978.

9. Marie-José Mondzain, Homo Spectator, Paris, Broché, 2013.

10. Henri Bergson, L'évolution créatrice, Paris, PUF, 2013.

11. Gilles Deleuze, Différence et répétition, Paris, PUF, 2011.

12. Friedrich Nietzsche, Le Crépuscule des Idoles, Paris, Broché, 2017.

13. Jacques Derrida, De la Grammatologie, op.cit.

\section{ABSTRACTS}

Abstract

We will question spatio-temporal writing as a means of articulating the visible and the sensible and as a potential for creating the possibility of subjectivation of the spectator when receiving a work of art. We will see what are the possible uses of current technologies in order to propose

original spectatorial experiences that presuppose a liberation from the codes of classical scenarios and a linear articulation of the senses that is a necessary condition for granting the term novelty in a creation.

Résumé

Nous questionnerons l'écriture spatio-temporelle comme moyen d'articuler le visible et le sensible et comme potentiel pour créer la possibilité d'une subjectivation du spectateur lors de la réception d'une œuvre d'art. Nous verrons quels sont les usages possibles des technologies actuelles afin que celles-ci proposent des expériences spectatorielles originales supposant de s'affranchir des codes des scénarii classiques et d'une articulation linéaire des sens qui est une condition nécessaire pour accorder le terme de nouveauté dans une création.

\section{AUTHOR}

\section{EDWIGE ARMAND}

Enseignante-Chercheuse à l'Institut National Polytechnique de Purpan, chercheuse associée au Laboratoire LARA-Seppia, Présidente de l'association Passerelle Art-Science-Technologie, a contribué à l'ouvrage collectif Dir. X.Lambert, Les enjeux cognitifs de l'artefact 
esthétique (Broché, 2019) et à la revue Dir. Y.C Zarka, Cités, Aujourd'hui le Posthumain ( $\mathrm{N}^{\circ} 55$ 2013). 